\title{
BioMEMS for sensing in cell characterization, drug screening and biofilm detection
}

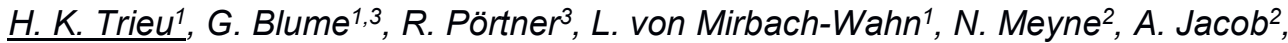 \\ D. Venegas Rojas ${ }^{1}$, M. Jücker ${ }^{4}$,K. Kalaydzhyan ${ }^{1}$ \\ 1 Inst. of Microsystems Technology, TUHH, Eissendorfer Str. 42, 21073 Hamburg, Germany, \\ 2 Inst. of High Frequency Technology, TUHH, Denickestr. 22, 21073 Hamburg, Germany \\ 3 Inst. of Bioprocess and Biosystems Engineering, TUHH, Denickestr. 15, 21073 Hamburg, Germany \\ ${ }^{4}$ Inst. of Biochemistry and Signal Transduction, UKE, Martinistr. 52, 20251 Hamburg, Germany \\ trieu@tuhh.de
}

\begin{abstract}
:
BioMEMS as microsystems designed for biomedical and biological applications provide various functions from micro implants, Lab-on-chip, PoC diagnostics to biosensors. This paper focuses on the sensing capability of BioMEMS in cell characterization, drug screening and biofilm detection. In situ staining and electrical approaches as impedance spectroscopy, dielectric spectroscopy and contactless impedimetric sensing are presented and discussed for applications in 3D cell culturing, for drug screening, sedimentation analysis in aquatic research and biofilm detection in indwelling devices.
\end{abstract}

Key words: BioMEMS, in situ staining, impedance spectroscopy, dielectric spectroscopy, impedimetric biofilm detection

\section{Introduction}

Micro-Electro-Mechanical Systems (MEMS) with feature size in the submillimeter down to micrometer range are well suited for the handling and interaction with living tissue and cells which correspond in geometry. Over the past few decades, this capability has enabled the exploration and exploitation of a novel biomedical engineering area called BioMEMS. Especially the integration of microfluidics, allowing the handling of samples in the order of microliters and nanoliters, makes these devices very popular for use in diagnostics. Examples of MEMS designed for biomedical and biological applications are micro implants, Lab-on-chip, Point of Care (PoC) diagnostics devices or biosensors.

This paper focuses on the sensing capability of BioMEMS. Sensing cell growth and viability in microfluidic bioreactors provides important parameters that should be addressed during every cultivation. There are different methods for the determination of cell number and viability. Offline methods like cell counting after viability staining with Trypan blue have the disadvantage that a sample has to be taken for the determination of cell number. The cultivation is disturbed and the number of samples is limited. Several stains for viability, like Acridine orange, intercalate with the DNA or are cytotoxic and can hence only be used after taking a sample from the reactor. For online monitoring of cell viability, the Calcein-AM (acetoxy methyl ester) stain can be used as this dye does not intercalate with the DNA and is low toxic. Microfluidic systems offer the possibility to apply the Calcein-AM staining solution in situ in the bioreactor. The cell viability can be monitored for several days via fluorescence microscopy. However, the intensity of the Calcein-AM stain decreases with every cell cleavage and after about 10 days, fluorescence cannot be observed anymore. Repetition of the staining procedure is required. Alternative approaches for online monitoring of cell growth and viability are electrical methods like impedance spectroscopy or dielectric spectroscopy. With MEMS, electrodes can be easily integrated in the microbioreactor enabling in situ sensing without disturbing the cells during the measurements. Moreover, there is no limitation by sample volume or number as in offline methods and measurement time is not limited as with the Calcein-AM stain. In the following, the in situ staining with Calcein-AM and electrical approaches as impedance spectroscopy, dielectric spectroscopy and contactless impedimetric sensing are presented and discussed for applications in 3D cell culturing, for drug screening, sedimentation analysis in aquatic research and biofilm detection in indwelling devices. 


\section{Microfluidic bioreactor for 3D cell culture and drug screening of hepatocellular carcinoma tumoroids}

The in situ staining approach with Calcein-AM has been adopted for a bioreactor cultivating tumoroids for drug screening [1]. Hepatocellular carcinoma (HCC) represents the second largest contributor to cancer mortality worldwide. Currently, there is no curative treatment for advanced stage HCC, so development of new therapies is urgently required. However, testing new drugs in standard 2D cell culture does not reflect the effect of this drug on a complex structure of a 3D tumor. Therefore, the objective of this project is to develop a novel microfluidic bioreactor capable of cultivating 3D tumoroids, as in vitro models, for drug screening research. A capture system for the tumoroids has been designed with the microfluidic bioreactor consisting of micropillars which also allows continuous supply with medium and drugs (see Fig. 1). Computational Fluid Dynamics was used to understand the effect of parameters such as flow velocity and shear stress. In order to avoid cell attachment to the bottom surface, HepG2, Hep3B, and Huh-7 cell lines were cultured on polydimethylsiloxane. Biocompatibility was confirmed and HepG2 cells were successfully grown as spherical tumoroids (see. Fig. 2), which remained healthy and viable for more than a week in the microbioreactor. Furthermore, the design enables in situ viability assays and continuous monitoring for days by using CalceinAM staining followed by fluorescent microscopy.

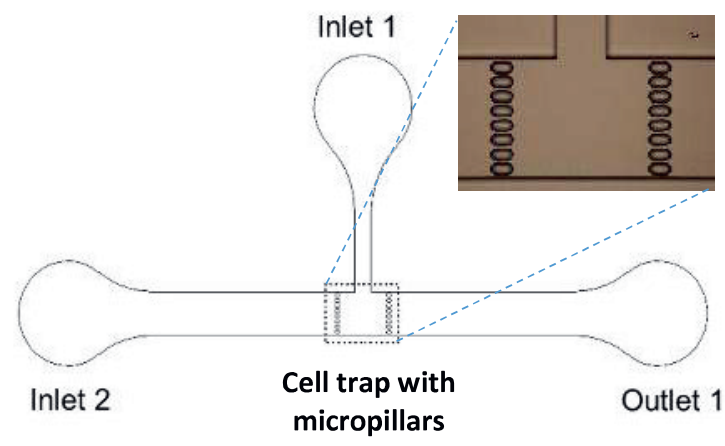

Fig. 1. Design of the microbioreactor.
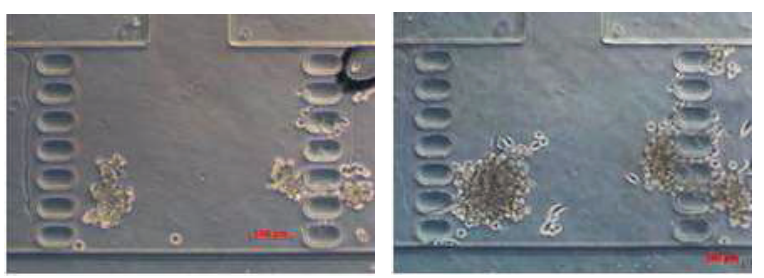

Fig. 2. HepG2 Tumoroids inoculation (left), and growing in the microbioreactor after 3 days (right).

The microbioreactor is made of PDMS and glass, so it provides a very good transparency for fluorescence spectroscopy (see Fig. 3). Besides, the quantity of stain necessary in the microbioreactor is in the order of microliters, unlike the standard procedures where several milliiters are necessary, reducing considerably the costs.

For staining the perfusion is stopped and the dye, Calcein AM in this case, is inserted through the outlet. The micropillars allow filling the chamber completely without wash-out of the cells as they keep the tumoroids in place. After $30 \mathrm{~min}$ incubation at $37{ }^{\circ} \mathrm{C}$ the perfusion is started in order to wash out the excess of the dye. The viability of the cells can now be monitored by fluorescence microscopy for days without disturbing the culture.
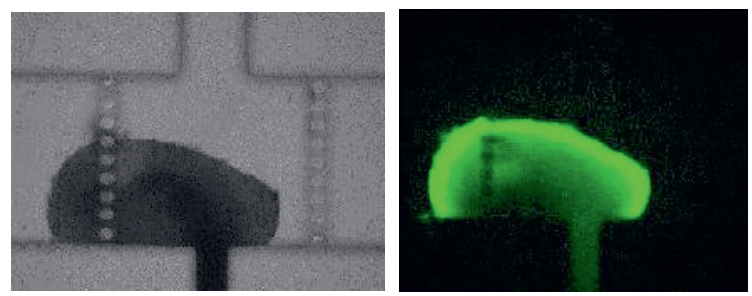

Fig. 3. HepG2 Tumoroids in the microbioreactor, captured with visual light (left) and with fluorescence microscopy (right).

\section{Impedance spectroscopy}

Tissue-like 3D cultures are a powerful tool for drug development and substance testing, mimicking the behavior of organs and living organisms. Online monitoring of cell viability and cell growth is essential. Impedance spectroscopy has made considerable progress and is an appropriate sensing technique. Cell viability can be determined by the so-called $\beta$-polarization relaxation measured in the range of a few $\mathrm{MHz}$ which corresponds to an intact cell membrane. This technique is also of interest in aquatic research where the organic compound in sedimentation is investigated by a sensor network.

The effect of the $\beta$-polarization and its relaxation, as well as its suitability for the determination of cell growth and viability, are briefly explained next. Cells have an overall negative charge, hence in an electrolyte, such as cell culture medium, extracellular cations are attracted by electrostatic forces and a double layer around the cell is formed. When an electric field is applied, intracellular cations and anions move towards the cathode and anode respectively, until reaching the cell membrane. An electric field inside the cell is established and the extracellular ions are attracted corresponding to their charge. These layers store charge electrostatically and a counter electric field is formed. When the frequency of the applied electric field increases beyond a certain magnitude, the movement of the ions is no longer able to follow this polarity change. The polarization relaxation occurs. The ions cannot 
form a counter electric field and hence contribute to the conductivity of the electrolyte. This effect is called $\beta$-polarization relaxation and due to the fact that only viable cells have an intact membrane where polarization can occur, it can be used for viability measurements of cells.

\section{Impedance sensors for online continuous measurement in $3 \mathrm{D}$ cell culture}

The objective of this project is the development of a scaffold with integrated sensors as a tool for continuous 3D-cell culture with online-monitoring of cell viability and cell growth [2]. A process for the fabrication of the scaffold with non-cytotoxic polymers has been developed in thin film technology. The frame of the scaffold consists of two polymers with different stiffness (polyimide and SU8-100), hence making it foldable. The separation walls that will be inserted into the frame contain electrodes on a polymer matrix in such a way that measurements at distinct positions in the scaffold are possible (see Fig. 4).

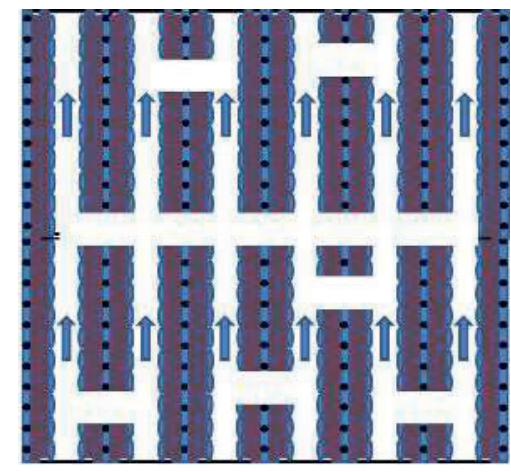

Fig. 4. Sketch of the polymer scaffold (blue) with integrated sensors (black) and cells (red).

In order to establish the impedance sensor, a second electrode design for measurements in 2D-cell culture in a 24-well-plate based bioreactor is developed and fabricated (see Fig. 5).

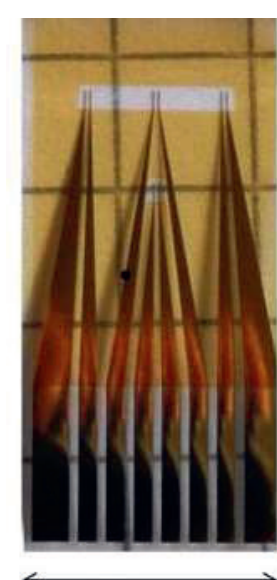

$10 \mathrm{~mm}$

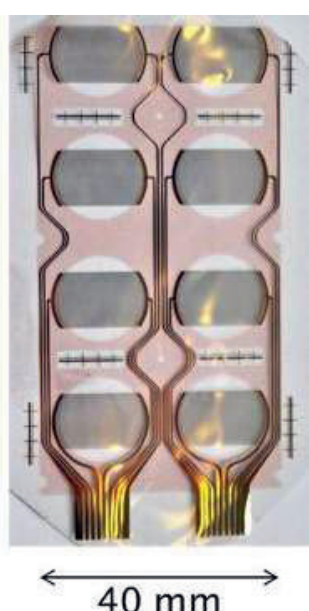

$40 \mathrm{~mm}$
Fig. 5. Two different electrode designs: Integrated electrode for the scaffold (left), interdigitated electrode for a 24-well-plate based bioreactor (right).
In order to characterize the electrodes, measurements of the electrode impedance have been conducted. The gold electrodes are coated with PEDOT (Poly-3,4-ethylendioxythiophen) which lowers the impedance (see Fig. 6).

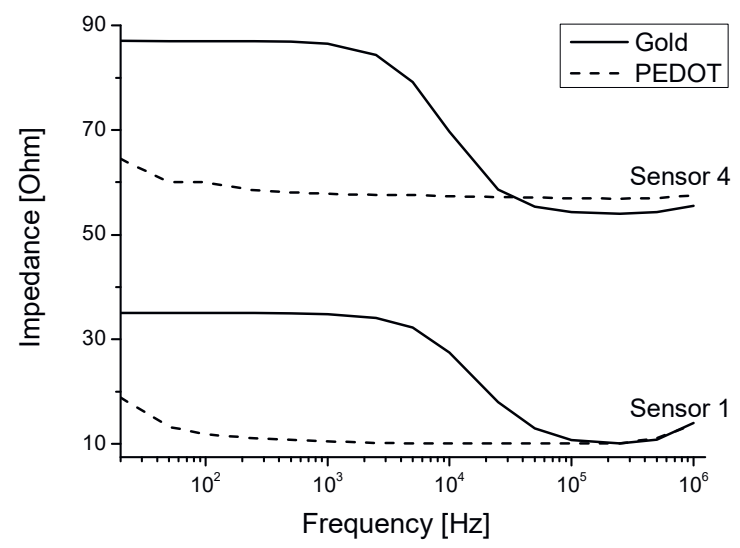

Fig. 6. Comparison of the electrode impedance prior to and after coating with PEDOT, measured for two electrode pairs indicated as sensor 1 and sensor 4 .

Cell growth can be monitored by the increase of the impedance. When cells are dead, their cell membrane disintegrates. Therefore, no $\beta$-polarization is possible and in consequence, there is no relaxation. This allows the discrimination between live and dead cells. Taking variations in the electrode impedance into account, a normalized impedance is used. This quantity is defined according to [3] as the ratio of the difference of the impedance measured during cell cultivation and the impedance obtained from the culture medium prior to the addition of cells with respect to the latter as a reference.

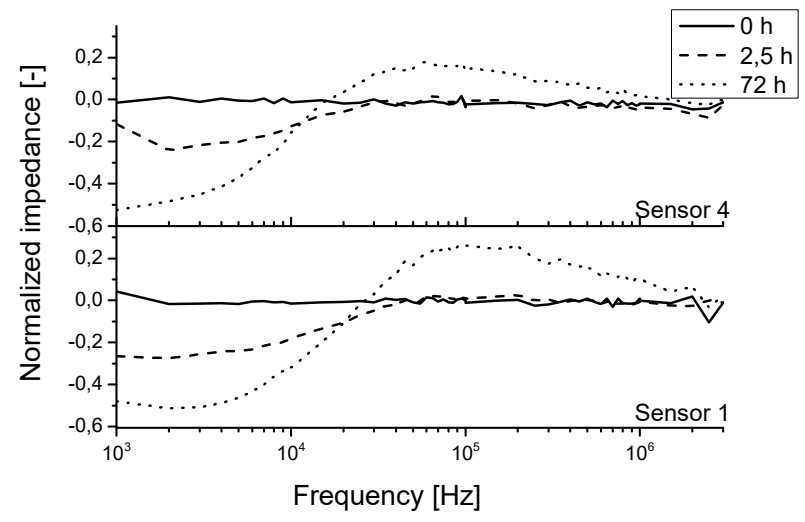

Fig. 7. The normalized impedance measured during cell cultivation over 3 days for two electrode pairs indicated as sensor 1 and sensor 4.

The cell growth can be clearly monitored as a function of time (see Fig. 7). After addition of cells in the culture medium, the impedance decreases at lower frequencies up to $2 \times 10^{4} \mathrm{~Hz}$. In literature, this effect is assumed to be linked to an increase in ionic concentration resulting from cell metabolism [3], [4]. 72 hours after 
starting cultivation, a significant relaxation in the frequency range of $10^{5}$ to $10^{6} \mathrm{~Hz}$ becomes obvious. This correlates well to the relaxation of the $\beta$-polarization reported in the literature between $10^{5}$ and $10^{7} \mathrm{~Hz}$ [5].

\section{Impedance sensors for in situ sedimentation analysis in aquatic research}

In aquatic research, reliable in situ information about organic compounds in sedimentation is still not available. A standard method is the measurement of the optical density of the fluid and the calculation of the statistical amount of living cells. This approach has some disadvantages as it does not allow to distinguish between particulate non-organic, living and dead organic compounds. At this point, the relaxation of the $\beta$-polarization again provides an appropriate solution. In order to measure the impedance of a fluid in situ, with a device embedded in a sensor network for long-term measurement, a low power electronics was developed with a 4point electrode arrangement and a single sinusoidal excitation [6]. The system consists of four different blocks, electrodes, excitation signal source, signal amplitude detector and the ADconverter. The sinusoidal signal is generated by a DDS between $1 \mathrm{kHz}$ and $20 \mathrm{MHz}$. The electrodes are made of ENEPIG (Electroless Nickel Electroless Palladium Immersion Gold) on Printed Circuit Board (PCB). The surface is coated with PEDOT for improved charge transfer characteristics. In a 4-point electrode arrangement the current flows through two electrodes and the voltage drop in the fluid is measured across the other two electrodes. Influence of unpredictable and unstable electrode or electrolyte effects on the measurement can be reduced with this arrangement. The voltage drop is measured by a logarithmic amplifier, which is sensitive to the amplitude of a signal and independent of the frequency. A second logarithmic amplifier measures the current through a known resistor. The amplitude of the sinusoidal signal is logarithmically converted by the logarithmic amplifier into a DC value. Both quantities are analog-digital converted by a differential ADC.

In order to measure the sedimentation of cells, the $\beta$-relaxation has to be detected regardless of the amount of other particles. As a proof of principle, one million cells with a diameter of 10 to $12 \mu \mathrm{m}$ were mixed with $4 \mathrm{ml}$ of nutrient solution and $1 \mathrm{~g}$ of sediment, which was autoclaved before. A second reference experiment was conducted without cells. A container with the electrodes at the bottom was filled with the cellsediment mixture and the solely sediment respectively. A measurement was performed every minute. A comparison of the results from both experiments clearly proves the impact of the presence of the cells on the relaxation of the $\beta$-polarization (see Fig. 8 and Fig. 9).

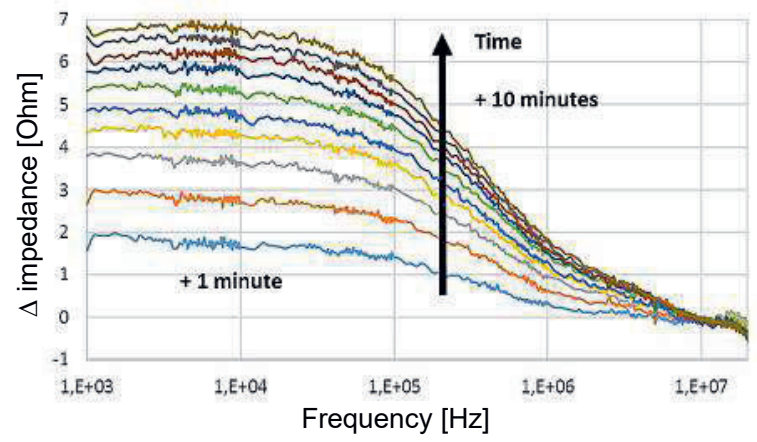

Fig. 8. Cell-sediment experiment: Impedance of the sedimentation process of the cell-sediment mixture over time with one measurement every minute.

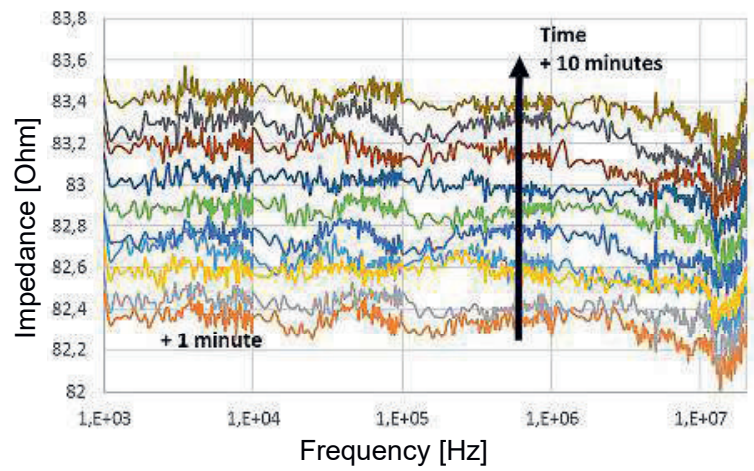

Fig. 9. Reference experiment: Impedance of the sedimentation process of the solely sediment over time with one measurement every minute.

The $\beta$-relaxation is visible at the transition between the low frequency (up to $10^{5} \mathrm{~Hz}$ ) and high frequency range $\left(10^{7} \mathrm{~Hz}\right)$ when the difference of the impedance between the initial and every following measurement of the cellsediment mixture is depicted against the frequency (see Fig. 8). For the reference experiment, without cells no relaxation is observed. The slight increase of the impedance over time reflects the impedance change caused by the particulate non-organic sediment (see. Fig. 9). With the detection of the $\beta$-relaxation it is possible to measure the sedimentation rate of living cells and to analyze the total amount of living cells in a certain volume of water in situ.

\section{Dielectric spectroscopy}

Novel dielectric sensors in the microwave frequency range have been proposed recently for non-invasive characterization of biological liquids and cells [7]. The basis for the dielectric spectroscopy of biological tissues is the difference of the dielectric permittivity of biological samples depending on the material composition. A miniaturized transmission-line sensor based on a coplanar waveguide (CPW) is used to characterize the permittivity of nanoliter volumes of biologically relevant liquids 
and cell suspensions [8]. The sensitivity of broadband CPW sensors is increased by means of a serpentine center conductor. The biological samples are supplied by a microfluidic channel across the sensor (see Fig. 10). The MEMS device is fabricated using a borosilicate glass wafer as a substrate material. The electrodes are patterned by etching a sputter-deposited metal layer (approx. $75 \mathrm{~nm}$ titanium and $625 \mathrm{~nm}$ gold). The walls of the channel are $200 \mu \mathrm{m}$ thick and made of SU-8 (MicroChem). An acrylic glass plate is glued to the SU-8 walls to form a closed channel. The inlet and outlet for liquid samples are realized using silicone tubing.

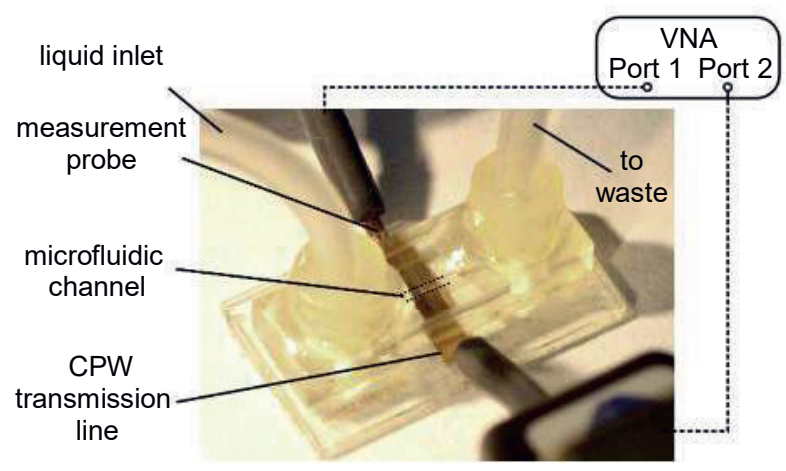

Fig. 10. Setup of the coplanar waveguide sensor with integrated microfluidic channel. [8]

The sensor operates between $850 \mathrm{MHz}$ and 40 $\mathrm{GHz}$ detecting the broadband permittivity of liquid samples such as aqueous salt and protein solutions. The different signatures of living and dead Chinese hamster ovary cells in suspension have been investigated. For numerical analysis, the difference of the complex relative permittivity $\varepsilon_{r}=\varepsilon_{r}^{\prime}-j \cdot \varepsilon_{r}^{\prime \prime}$ is determined and expressed as contrast $\Delta \varepsilon_{r}=\Delta \varepsilon_{r \text {,medium }}-\Delta \varepsilon_{r \text {,suspension. The }}$ experimentally detected contrast between living and dead Chinese hamster ovary cells in suspension is small, but significant, despite the small nanoliter sample volume and low cell concentrations. Detailed data are provided in [8].

The contrast is slightly smaller for the dead cells, which is in agreement with results from literature [9]. The biological explanation for this is that the cell membrane starts to disintegrate after cell death, leading to an increased exchange between the cytoplasm and the culture medium. In the case of cell membrane disintegration, ions and proteins diffuse from the cytosol to the protein-free culture medium, leading to a local change in concentration. Additionally, many proteins are degraded or denatured during cell death and lose their biochemical features along with their secondary to quaternary structure.

All these mechanisms potentially affect the dielectric contrast. Most likely, it represents the superposition of several effects.

In [8] exemplary measurements of solutions of the protein albumin are performed. The spectrum of the detected contrast between different protein concentrations corresponds well with that of the contrast between living and dead cell suspensions. This suggests that part of the dielectric contrast is due to local changes of protein concentration.

\section{Contactless impedimetric biofilm detection}

Last but not least, this paper addresses the topic of biofilm detection using contactless microfluidic impedimetric measurement. Biofilms encounter at any interface of biofluids and technical solid surfaces. Indwelling catheters are associated with very high risk of colonization by the bacteria originated from patients, healthcare personnel or other sources in the environment. There is a need of online accessible information about the bacteriuria in the catheter allowing the implementation of an effective procedure for the replacement of catheter. In order to sense the development of biofilms without immediate physical contact of the electrode material with the biofluids in-vivo, a contactless impedimetric sensing approach is proposed. Hereby, two coplanar electrodes are used, coated with a thin electrical insulating film. Alternating current (AC) is applied to the electrodes, and the complex impedance response of the setup is measured. When a biofilm attaches to the surface of the electrode coating, the impedance will change due to the fact that the biofilm behaves differently than the biofluid and therefore the impeding current flow between the electrodes is altered.

During the first 2 days, the proliferated bacteria formed a biofilm that could be visually observed as specific contrast surface relief (see Fig. 11). The bacteria were embedded into the biofilm and being active, moving within their wells in the biofilm.

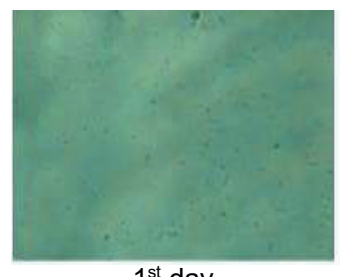

$1^{\text {st }}$ day

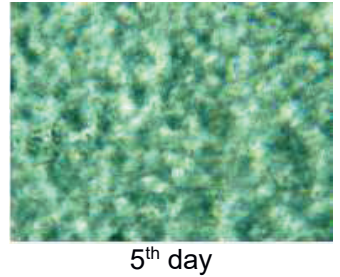

Fig. 11. Biofilm development on the channel surface over 6 days.
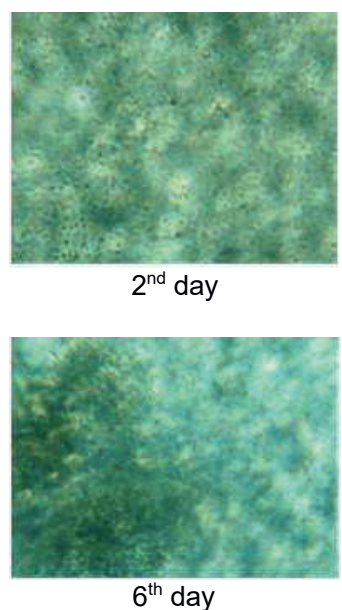
To the $5^{\text {th }}$ day the biofilm was visually maturated and beginning this day, abrupt fall of the bacteria mobility was observed. At the $6^{\text {th }}$ day, big fragments of the biofilm with non-mobile bacteria inside detached and flowed through the channel. The bacteria were optically hardly distinguishable. Their size was reduced. They were not active and probably not alive. The abrupt changes occurred during just one day.

A compact handheld device was built to sense the development of the biofilm in the channel at $100 \mathrm{kHz}$. The output signal followed the biofilm growth and death (see Fig. 12a) that was proved by an impedance analyzer (see Fig. 12b).
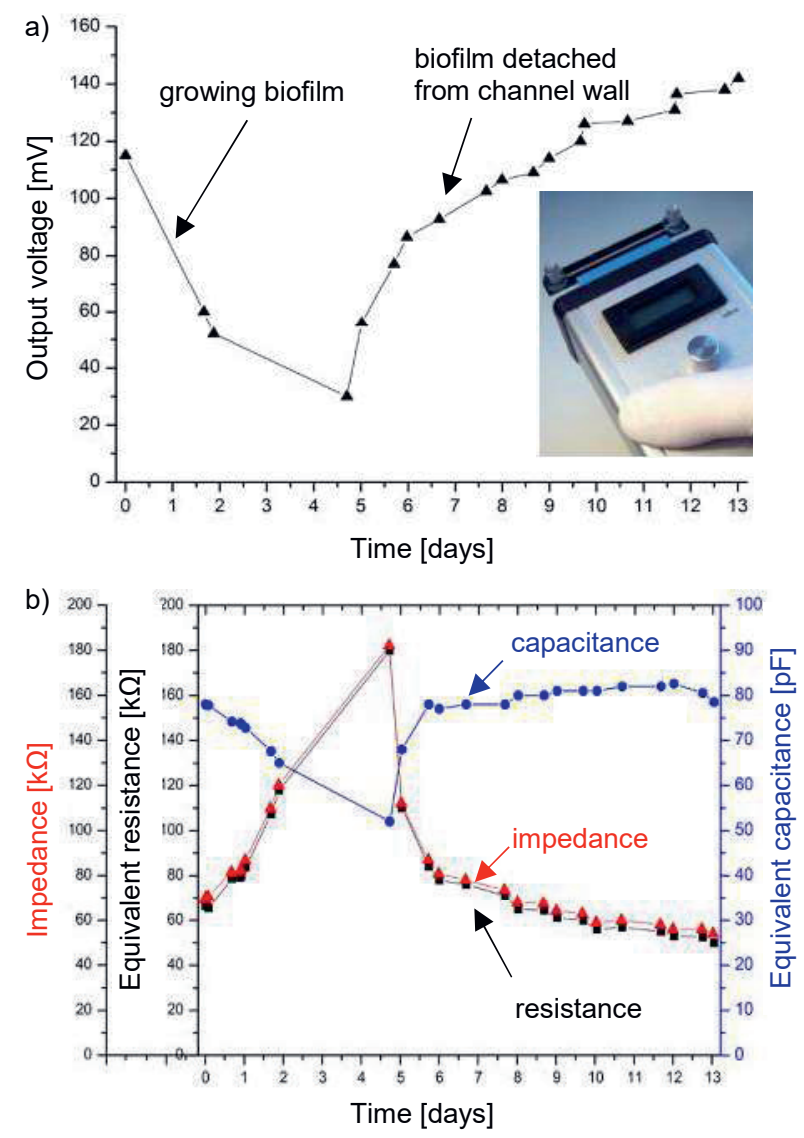

Fig. 12. Temporal signal change in the course of the biofilm development in a microfluidic channel. Output from handheld device (a), reference output from impedance analyzer (b).

In the present approach, the sensitivity of biofilm detection was defined not only by the electric properties of the biofluids and biofilms, but also by the microfluidic channel and electrode geometries providing the possibility of further design optimization for enhanced performance.

\section{Acknowledgement}

The projects "Charakterisierung der Zellwachstumsaktivität von Gewebezellen in 3D-Scaffolds mit integrierten Sensoren" and "Multiparameter-
Sensor-Mikrosystem für die Meeresforschung“ are funded by the German Research Council (DFG).

The project „Tumoroids in micro-bioreactors for screening drugs against hepatocellular carcinoma (HCC) is financially supported by Forschungszentrum Medizintechnik Hamburg.

\section{References}

[1] D. Venegas-Rojas, D. Nörz, F. Ewald, M. Jücker, H.K. Trieu, Microfluidic bioreactor for 3D Cell Culture and Drug Screening of Hepatocellular Carcinoma Tumoroids, EMBL Conference Microfluidics 2016. Heidelberg, Germany.

[2] G. Blume, L. von Mirbach-Wahn, G. Mielke, H.K. Trieu, R. Pörtner, Impedance Sensors and Scaffold for 3D Tissue Culture, 3D Cell Culture 2016: How close to 'in vivo' can we get? Models, applications \& translation. Freiburg, Germnay.

[3] X. Huang, D. Nguyen, D.W. Greve, M.M. Domach, Simulation of Microelectrode Impedance Changes Due to Cell Growth. IEEE Sensors Journal, 2004, 4(5):576-583. DOI: 10.1109/JSEN.2004.831302

[4] X. Cheng, Y. Liu, D. Irimia, U. Demirci, L. Yang, L. Zamir, W.R. Rodriguez, M. Toner, R. Bashir, Cell detection and counting through cell lysate impedance spectroscopy in microfluidic devices. Lab Chip, 2007, 7(6):746. DOI: $10.1039 / \mathrm{b} 705082 \mathrm{~h}$

[5] N.F. Sheppard, M. Eden, G. Kantor, 1994. Proceedings of the 16th Annual International Conference of the IEEE Engineering in Medicine and Biology Society. Engineering advances, new opportunities for biomedical engineers Baltimore, Maryland, USA, November 3-6, 1994.

[6] L. Wahn, T. Gentz, H.K. Trieu, M. Schlüter, J. Müller, Multi-Parameter Low-Power Sensor Network for Aquatic Research, Proceedings SENSOR 2015, pp. 647-650, DOI: 10.5162/sensor2015/D8.2

[7] K. Grenier, D. Dubuc, P.E. Poleni, M. Kumemura, H. Toshiyoshi, T. Fujii, H. Fujita, Integrated broadband microwave and microfluidic sensor dedicated to bioengineering, IEEE Transactions on Microwave Theory and Techniques, vol. 57, no. 12 , pp. 3246-3253, Dec. 2009. DOI: 10.1109/TMTT.2009.2034226

[8] N. Meyne née Haase, G. Fuge, H.K. Trieu, A.P. Zeng, A.F. Jacob, Miniaturized Transmission-Line Sensor for Broadband Dielectric Characterization of Biological Liquids and Cell Suspensions, IEEE Transactions on Microwave Theory and Techniques, vol. 63, no. 10, pp. 3026-3033, Oct. 2015, DOI: 10.1109/TMTT.2015.2472009

[9] F. Artis, D. Dubuc, J.J. Fournie, M. Poupot, K. Grenier, Microwave dielectric spectroscopy of cell membrane permeabilization with saponin on human B lymphoma cells, IEEE MTT-S Int. Microw. Symp. Dig., Jun. 2014, pp. 1-4. DOI: 10.1109/MWSYM.2014.6848520 